\title{
Spin textures induced by quenched disorder in a reentrant spin glass: Vortices versus "frustrated" skyrmions
}

\author{
I. Mirebeau,,${ }^{1, *}$ N. Martin, ${ }^{1}$ M. Deutsch, ${ }^{2}$ L. J. Bannenberg, ${ }^{3}$ C. Pappas,${ }^{3}$ G. Chaboussant, ${ }^{1}$ R. Cubitt,${ }^{4}$ \\ C. Decorse ${ }^{5}$ and A. O. Leonov 6,7 \\ ${ }^{1}$ Laboratoire Léon Brillouin, CEA, CNRS, Université Paris-Saclay, CEA Saclay 91191 Gif-sur-Yvette, France \\ ${ }^{2}$ Université de Lorraine, CNRS, CRM2, Nancy, France \\ ${ }^{3}$ Faculty of Applied Science, Delft University of Technology, $2629 \mathrm{JB}$ Delft, The Netherlands \\ ${ }^{4}$ Institut Laue Langevin, BP156, F-38042 Grenoble France \\ ${ }^{5}$ ICMMO, Université Paris-Sud, Université Paris-Saclay, F-91405 Orsay, France \\ ${ }^{6}$ Chiral Research Center, Hiroshima University, Higashi-Hiroshima, 739-8526, Japan \\ ${ }^{7}$ Department of Chemistry, Faculty of Science, Hiroshima University Kagamiyama, Higashi Hiroshima, Hiroshima 739-8526, Japan
}

(Received 14 February 2018; revised manuscript received 27 June 2018; published 19 July 2018)

\begin{abstract}
Reentrant spin glasses are frustrated disordered ferromagnets developing vortexlike textures under an applied magnetic field. Our study of a $\mathrm{Ni}_{0.81} \mathrm{Mn}_{0.19}$ single crystal by small angle neutron scattering clarifies their internal structure and shows that these textures are randomly distributed. Spin components transverse to the magnetic field rotate over length scales of 3-15 $\mathrm{nm}$ in the explored field range, decreasing as field increases according to a scaling law. Monte Carlo simulations reveal that the internal structure of the vortices is strongly distorted and differs from that assumed for "frustrated" skyrmions, built upon a competition between symmetric exchange interactions. Isolated vortices have small noninteger topological charge. The vortices keep an anisotropic shape on a three-dimensional lattice, recalling "croutons" in a "ferromagnetic soup." Their size and number can be tuned independently by the magnetic field and concentration $x$ (or heat treatment), respectively. This opens an original route to understand and control the influence of quenched disorder in systems hosting nontrivial spin textures.
\end{abstract}

DOI: 10.1103/PhysRevB.98.014420

\section{INTRODUCTION}

Disorder plays a central role in the advent of the most spectacular quantum phenomena observed in condensed matter. The quantum Hall effect observed in a two-dimensional (2d) electron gas [1,2], the two-current character of the resistivity in impurity-containing ferromagnetic metals [3] leading to giant magnetoresistance [4], or the dissipationless conduction observed in the mixed state of type-II superconductors [5,6] are prominent examples. Frustrated ferromagnets represent another type of playground to study the influence of disorder. Such systems show competing ferromagnetic (FM)/antiferromagnetic (AFM) interactions combined with atomic disorder. The influence of quenched disorder, when treated in a mean-field model with infinite range interactions $[7,8]$, leads to a canonical spin glass (SG) when the average interaction $\bar{J}$ is smaller than the width of the interaction distribution or to a reentrant spin glass (RSG) otherwise. Here, we focus on the FM case $(\bar{J}>0)$ of the RSGs where vortexlike textures are stabilized under an applied magnetic field at low temperature. We study their morphology and spatial organization by combining neutron scattering experiments on a $\mathrm{Ni}_{0.81} \mathrm{Mn}_{0.19}$ single crystal and Monte Carlo simulations. We compare them with those expected for skyrmions built upon a competition between symmetric exchange interactions. Altogether, our study shows that one can independently tune

\footnotetext{
*isabelle.mirebeau@cea.fr
}

the number and size of vortex textures in frustrated disordered magnets with the magnetic field, heat treatment, and concentration of magnetic species. It provides clues to control and use the influence of quenched disorder in frustrated ferromagnets and skyrmion-hosting systems in bulk state.

\section{REENTRANT SPIN GLASSES AND "FRUSTRATED" SKYRMIONS}

As a common feature, RSGs show three successive phase transitions upon cooling: a paramagnetic to FM transition at $T_{C}$ followed by transitions towards two mixed phases at $T_{K}$ and $\mathrm{T}_{\mathrm{F}}$. Below the canting temperature $\mathrm{T}_{\mathrm{K}}$, spin components $\mathbf{m}_{\mathrm{T}}$ transverse to the longitudinal magnetization $\mathbf{m}_{\mathrm{L}}$ start to freeze. The lower temperature $T_{F}$ marks the onset of strong irreversibilities of $\mathbf{m}_{\mathrm{L}}$. In this picture, the ferromagnetic longrange order of $\mathbf{m}_{\mathrm{L}}$ is preserved in the RSG down to $\mathrm{T} \rightarrow 0 \mathrm{~K}$. The phase diagram $(\mathrm{T}, x)$, where $x$ is a parameter tuning the distribution of interactions, shows a critical line between SG and RSGs ended by a multicritical point at $x_{\mathrm{C}}$ where all phases collapse [8]. Metallic ferromagnetic alloys with competing nearest-neighbor interactions tuned by the concentration $x$ show a magnetic phase diagram (T, $x$ ) in qualitative agreement with mean-field predictions. Well-known examples are $\mathrm{Ni}_{1-x} \mathrm{Mn}_{x}$ [9], $\mathrm{Au}_{1-x} \mathrm{Fe}_{x}$ [10], $\mathrm{Fe}_{1-x} \mathrm{Al}_{x}$ [11,12], and $\mathrm{Fe}_{1-x} \mathrm{Cr}_{x}$ [13] crystalline alloys or amorphous $\mathrm{Fe}$-based alloys [14-18]. A large body of experimental and theoretical studies have revealed the peculiarities of their magnetic behavior. 
In this paper, we focus on vortexlike textures observed in the 1980 s in the above systems, either in single crystal, polycrystal, or amorphous form [12,19-21]. They were detected under applied magnetic field in the mixed phases of ferromagnetic, weakly frustrated alloys $\left(x \ll x_{\mathrm{C}}\right)$, using small angle neutron scattering (SANS), which provides a clear signature of these textures and reveals their typical size. Inside the vortices, the transverse spin components are frozen in the plane perpendicular to the applied field, and they are rotated over a finite length scale, yielding a maximum in the neutron scattering cross section versus the momentum transfer. In addition, the transverse spin freezing induces Dzyaloshinskii-Moriya (DM) anisotropy [22], together with a chiral anomalous Hall effect [23-25]. Stimulated by these measurements, Monte Carlo (MC) simulations were performed in a $2 \mathrm{~d}$ lattice, showing similar vortexlike patterns [26]. The knowledge of their spatial organization has, however, remained elusive.

In this context, it is worth recalling that ferromagnets may also host nanometric spin textures known as skyrmions (SKs). SKs form double-twist solitonic structures, offering many perspectives in spintronics and data storage [27,28]. As predicted by theory [29-33], some anisotropic ordered magnets with competing nearest neighbor $(\mathrm{NN})$ and next nearest neighbor (NNN) exchange interactions may host localized SKs with versatile internal structure and smooth rotation of the magnetization. Different types of modulated phases such as hexagonal or square SK lattices have been predicted, yielding a very rich phase diagram [30].

The size of these "frustrated" SKs, of the order of a few lattice constants, is comparable to the typical vortex size in RSGs and much smaller than the size of chiral SKs stabilized by DM anisotropy in thin films or bulk state, which is usually above $10 \mathrm{~nm}[34,35]$. Therefore quenched disorder should affect frustrated SKs much more than their chiral counterparts, expected to undergo a collective pinning by disordered impurities without deep changes of their internal structure [36]. Experimentally, large SK lattices were observed in noncentrosymmetric frustrated alloys with chemical disorder [37,38], showing magnetic anomalies similar to the RSG's. Frustrated SKs have been suspected in very few systems so far, such as $\mathrm{Gd}_{2} \mathrm{PdSi}_{3}$ (Ref. [39]).

Remarkably, frustrated SKs reveal strong similarities with the vortex textures observed in RSGs. Our study attempts to clarify the subtle differences between these two types of topological defects. To that end, we report on new experiments performed on a weakly frustrated $\mathrm{Ni}_{0.81} \mathrm{Mn}_{0.19}$ single crystal, searching for a vortex lattice and aiming for a better characterization of these field-induced magnetic textures (Sec. III). Our experiments are complemented by MC simulations with a minimal model, which clarifies the internal structure of the vortices and identifies their most relevant features (Sec. IV). We discuss the origin of the vortex textures, and compare them with SKs, either chiral or frustrated, observed in bulk materials (Sec. V).

\section{VORTEXLIKE TEXTURES IN A SINGLE-CRYSTALLINE REENTRANT SPIN GLASS}

\section{A. The $\mathrm{Ni}_{1-x} \mathrm{Mn}_{x}$ system and studied sample}

In $\mathrm{Ni}_{1-x} \mathrm{Mn}_{x}$ alloys, magnetic frustration arises from competing interactions between NN pairs, namely the AFM
Mn-Mn pairs and the FM Ni-Mn and Ni-Ni pairs $[43,44]$. The NNN Mn-Mn pairs are FM. The multicritical line between RSG and SG phases is located around $x_{\mathrm{C}}=0.24$, close to the stoichiometric $\mathrm{Ni}_{3} \mathrm{Mn}$ [see Refs. [40,41] and Fig. 1(a)]. Strikingly, the $\mathrm{Ni}_{3} \mathrm{Mn}$ ordered superstructure of $L 1_{2}$ type and space group $P m \overline{3} m$ eliminates all NN Mn-Mn pairs. This offers the possibility of tuning the magnetic order by controlling the number of such pairs through an appropriate heat treatment [45-47]. The fully ordered $\mathrm{Ni}_{3} \mathrm{Mn}$ is a ferromagnet with a Curie temperature $\mathrm{T}_{\mathrm{C}} \sim 450 \mathrm{~K}$, whereas a disordered alloy of the same composition (space group $F m \overline{3} m$ ) is a spin glass with a freezing temperature $\mathrm{T}_{\mathrm{F}} \sim 115 \mathrm{~K}$.

Here, we study a $\mathrm{Ni}_{0.81} \mathrm{Mn}_{0.19}$ single crystal, already used for the neutron scattering experiments presented in Ref. [21]. The single-crystal form limits the distributions of magnetocrystalline anisotropies and demagnetizing fields within the sample, and provides the best playground to search for a vortex lattice. A thin rectangular plate was cut from the large crystal in a (110) plane for magnetic measurements. Both samples were heated at $900{ }^{\circ} \mathrm{C}$ during 20 hours in a sealed quartz tube under vacuum, then quenched into an ice and water mixture to ensure maximal disorder [41]. They were stored in liquid nitrogen between experiments to prevent any further evolution of the short-range order.

Static magnetic susceptibility was measured versus temperature under a field $\mathrm{H}=20$ Oe in both field cooled (FC) and zero-field-cooled (ZFC) conditions, using a superconducting quantum interference device (SQUID). With decreasing temperature, the ZFC susceptibility strongly increases at the Curie temperature $\mathrm{T}_{\mathrm{C}}=257 \mathrm{~K}$, shows a plateau over an extended temperature range as expected for weakly frustrated RSGs, and then decreases [Fig. 1(b)]. The freezing temperature $\mathrm{T}_{\mathrm{F}}=18 \mathrm{~K}$, defined similarly to $\mathrm{T}_{\mathrm{C}}$ by the inflection point of the susceptibility versus temperature in the $\mathrm{ZFC}$ state, locates the onset of strong magnetic irreversibilities. The ratio $\mathrm{T}_{\mathrm{F}} / \mathrm{T}_{\mathrm{C}} \simeq 0.07$ characterizes the weak frustration of our sample. The canting temperature $T_{K} \sim 120 \mathrm{~K}$, which is situated between $T_{C}$ and $\mathrm{T}_{\mathrm{F}}$ locates much weaker irreversibilities related to transverse spin freezing. It was determined by previous neutron scattering experiments [20]. The three characteristic temperatures merge at the critical point.

\section{B. Small-angle neutron scattering}

SANS measurements were performed on the D33 instrument of the Institut Laue Langevin (ILL), using an incident neutron wavelength $\lambda=6 \AA$ and a sample to detector distance $\mathrm{D}=2.8 \mathrm{~m}$. Data were corrected for the detector efficiency and calibrated cross sections were obtained by taking the sample thickness and transmission, as well as the incident neutron flux, into account [48]. A magnetic field $\mathrm{H}$ up to $2 \mathrm{~T}$ was applied to the sample, in two configurations (see Fig. 2): (a) along the neutron beam, which defines the $y$ axis; (b) along the $x$ axis perpendicular to the neutron beam, namely, in a plane parallel to the detector $(x, z)$ plane. Additional measurements were performed in configuration (b) on the PAXY spectrometer of the Laboratoire Léon Brillouin (LLB) under a magnetic field up to $8 \mathrm{~T}$ for the same neutron wavelength and sample-to-detector distance. Figure 2 shows typical intensity maps recorded in the detector plane for the two configurations. The intensity is 


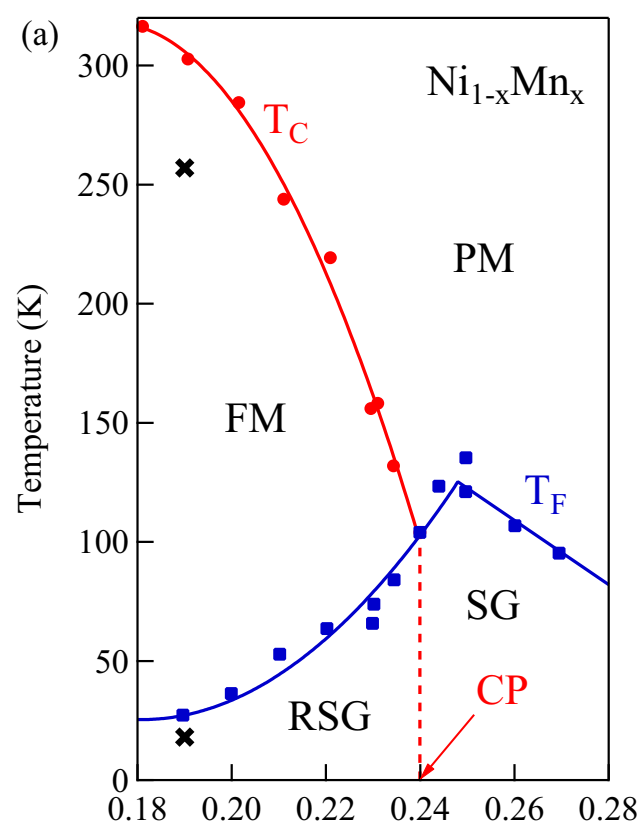

$\mathrm{X}$

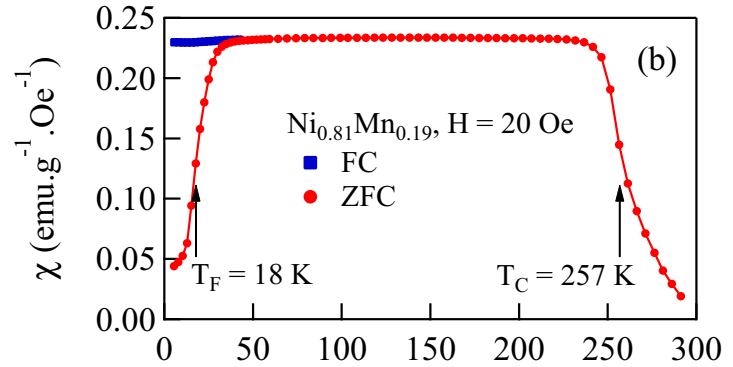

Temperature $(\mathrm{K})$

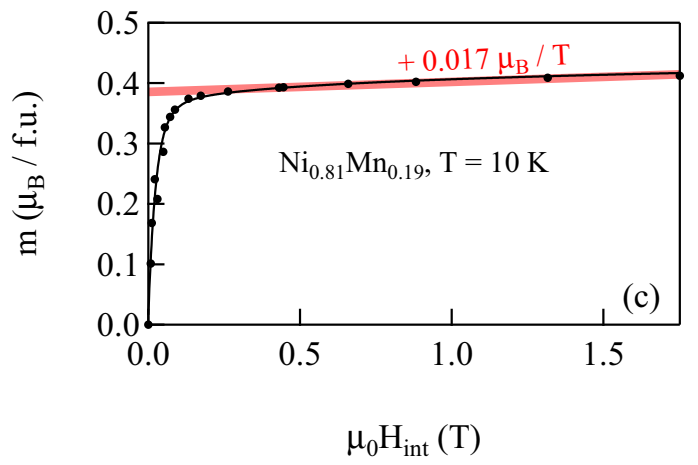

FIG. 1. (a) Magnetic phase diagram of the disordered $\mathrm{Ni}_{1-x} \mathrm{Mn}_{x}$ system determined by bulk magnetic measurements and adapted from Ref. [40]. A critical point (CP) occurs for $x \sim 0.24$, see Ref. [41]. (b) Static susceptibility $\chi$ of the $\mathrm{Ni}_{0.81} \mathrm{Mn}_{0.19}$ sample-initially, zero field cooled down to $4.2 \mathrm{~K}$-recorded upon warming under a magnetic field of 20 Oe (ZFC, red circles) then upon cooling under field (FC, blue squares). The characteristic freezing $\left(\mathrm{T}_{\mathrm{F}} \sim 18 \mathrm{~K}\right)$ and Curie $\left(\mathrm{T}_{\mathrm{C}} \sim 257 \mathrm{~K}\right)$ temperatures are obtained from the extrema of the temperature derivative of $\chi$ and reported in (a) (black crosses). (c) Field dependence of the magnetization of $\mathrm{Ni}_{0.81} \mathrm{Mn}_{0.19}$ (reproduced from Ref. [42]). After a quick rise, $m$ tends to saturate and further evolves with a small, yet finite, slope (red line).

measured at $3 \mathrm{~K}$ in the $\mathrm{ZFC}$ state under a magnetic field $\mathrm{H}=2 \mathrm{~T}$, which almost saturates the sample magnetization [Fig. 1(c)].

In configuration (a), the intensity distribution does not show any Bragg spot, rather a broad maximum at a finite momentum transfer. The intensity is isotropically distributed over a ring of scattering in the detector plane. The absence of any Bragg spots strikingly contrasts with the scattering patterns in SK lattices or superconducting flux line lattices observed in single-crystal samples for the same experimental configuration $[49,50]$. It means that although the sample is single crystalline, the magnetic defects are organized in a random or liquidlike way. As discussed below, this is due to the random occupation of the lattice sites and subsequent disorder of Mn-Mn NN AFM bonds.

In configuration (b), one observes a similar pattern, but the intensity is now modulated according to the orientation of the momentum transfer with respect to the applied field, and
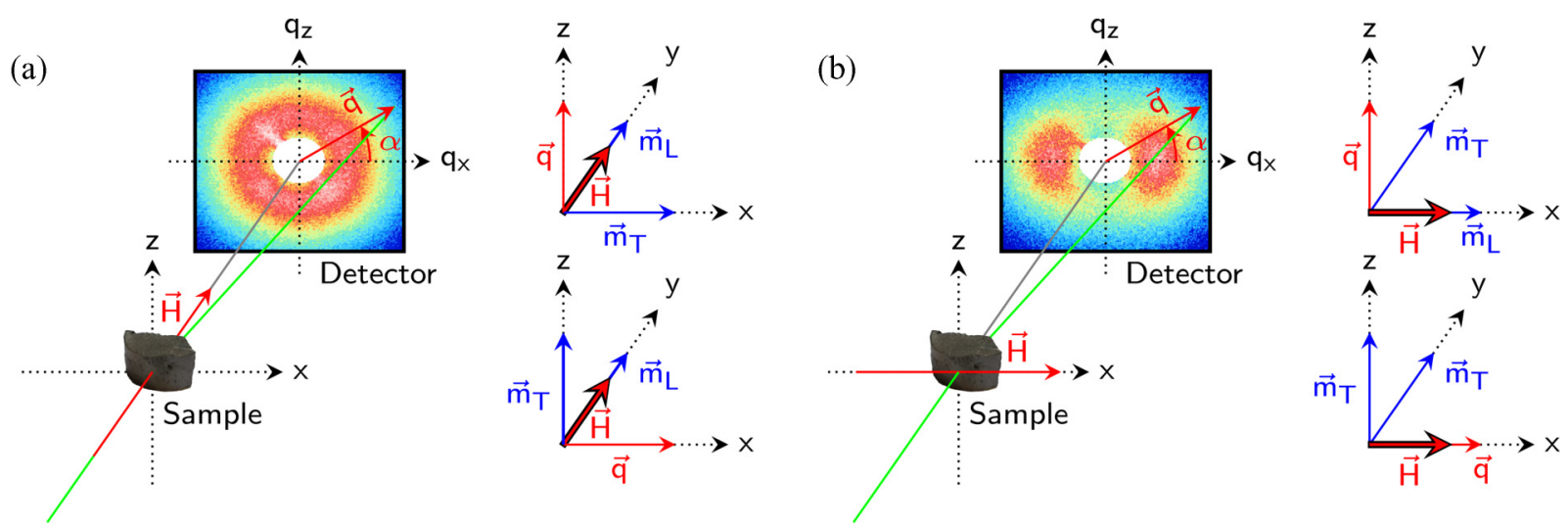

FIG. 2. Schematic configurations of the SANS experiments with the applied magnetic field $\mathbf{H}$ parallel to the neutron beam (a) and with $\mathbf{H}$ parallel to the detector (b). Orientations of the transverse $\mathbf{m}_{\mathrm{T}}$ and longitudinal $\mathbf{m}_{\mathrm{L}}$ spin components are shown in each case, which, combined to the selection rules of magnetic neutron scattering [Eq. (1)], leads to the corresponding patterns (here recorded at $\mathrm{T}=3 \mathrm{~K}$ and $\mathrm{H}=2 \mathrm{~T}$ after zero-field cooling). In both configurations, the magnetic field is applied along the [110] (or equivalent) crystallographic direction. 
(a)

$$
\mathrm{T}=3 \mathrm{~K}, \mathrm{H}=2 \mathrm{~T}
$$
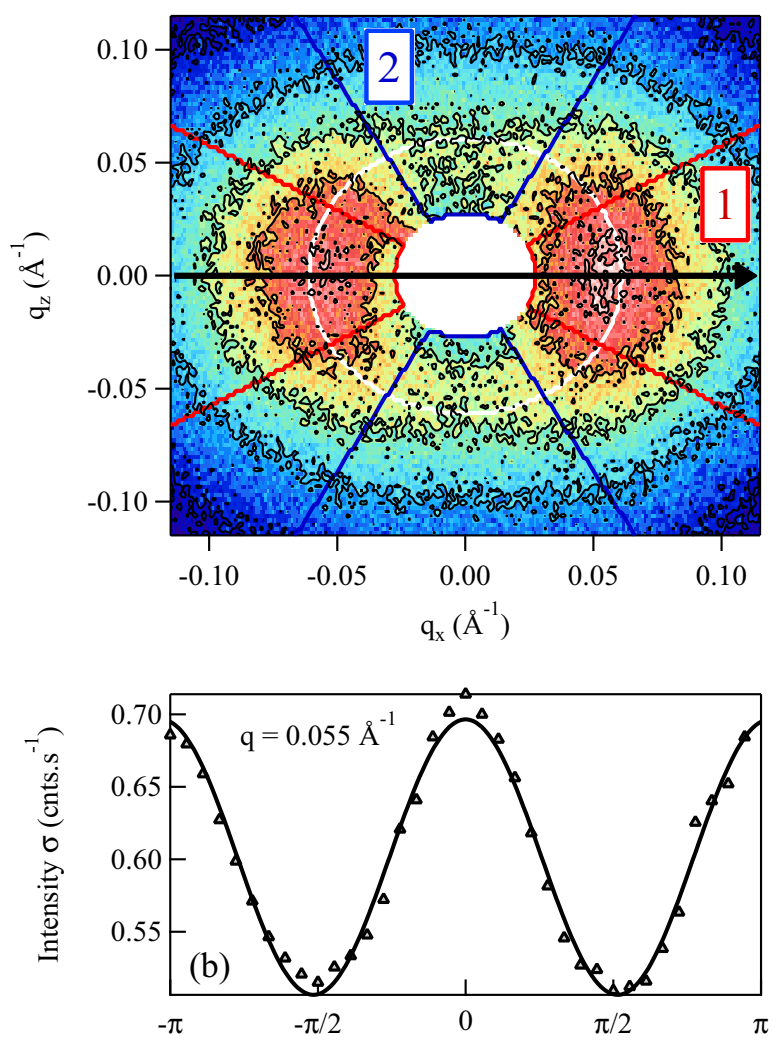

Azimuth $\alpha$
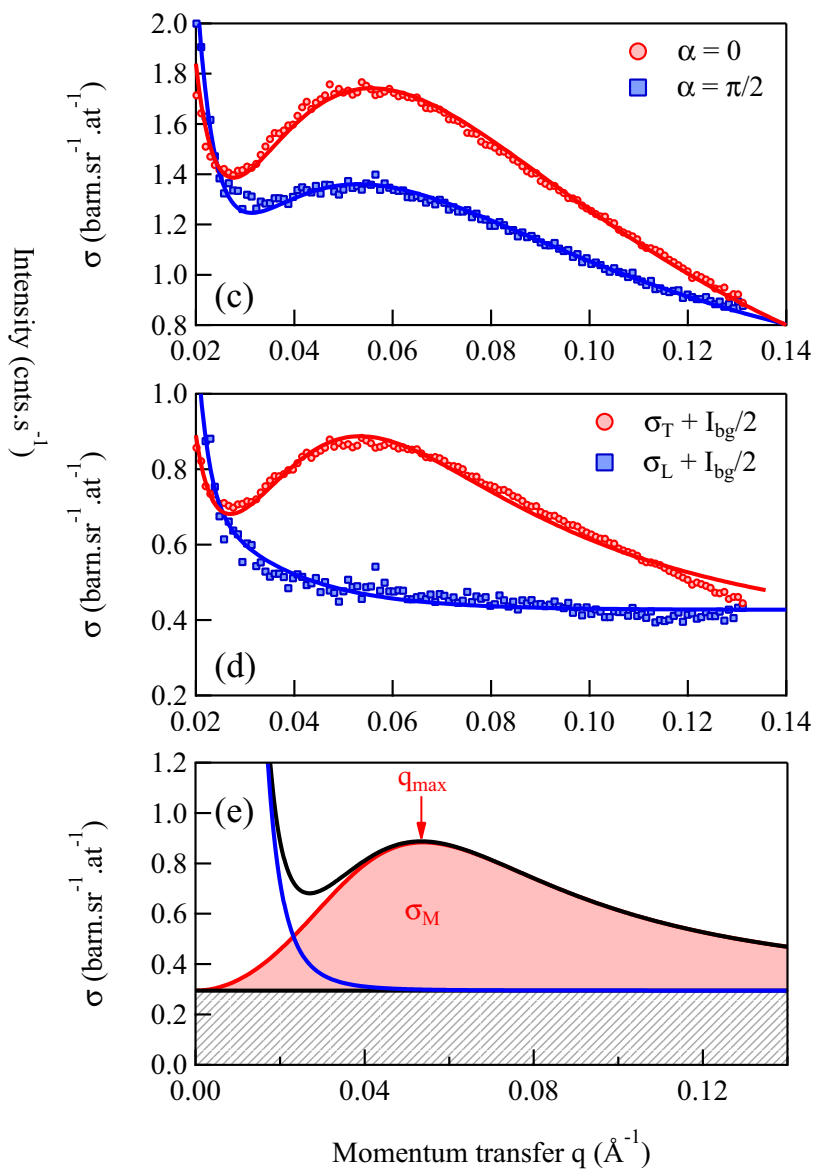

FIG. 3. (a) Scattering map recorded at $\mathrm{T}=3 \mathrm{~K}$ under a field $\mathrm{H}=2 \mathrm{~T}$ applied perpendicular to the incident beam (black arrow). The two $60^{\circ}$ angular ranges associated with $\mathbf{q} \| \mathbf{H}$ (red sector 1) and $\mathbf{q} \perp \mathbf{H}$ (blue sector 2) are also shown. (b) Angular dependence of the intensity at a momentum transfer $q=0.055 \AA^{-1}$ [i.e., collected along the white circular trace in (a)]. Solid line is a fit of Eq. (1) to the data. (c) $q$ dependence of the scattering cross section for momentum transfers along (red circles) and perpendicular (blue squares) to the applied field. Solid lines with corresponding colors are fits of Eq. (2) to the data. (d) $q$ dependence of the longitudinal $\left(\sigma_{\mathrm{T}}\right)$ and transverse $\left(\sigma_{\mathrm{L}}\right)$ magnetic cross sections, obtained by linear combinations of Eq. (1) for $\alpha=0(\mathbf{q} \| \mathbf{H})$ and $\pi / 2(\mathbf{q} \perp \mathbf{H})$. (e) Fit curve corresponding to the $\sigma_{\mathrm{T}}(q)$ data of (d) (black line) along with a singled out peak function (red curve) and $q$-dependent background signal (blue curve). The $q$-independent background contribution is also shown (hatched area, see text and Ref. [48]).

is strongly enhanced in the direction $\mathbf{q} \| \mathbf{H}$. This modulation comes from the selection rules for magnetic neutron scattering, which impose that only the spin components perpendicular to the scattering vector $\mathbf{q}$ contribute to the magnetic crosssection. As schematically explained in Fig. 2, the dominant contribution to the scattering in this configuration arises from spin components $\mathbf{m}_{\mathrm{T}}$ transverse to the magnetic field. In the following analysis, we focus on this configuration, which allows us to better characterize the spin textures. The intensity maps in configuration (b) can be described as

$$
\sigma(q, \alpha)=\sigma_{\mathrm{L}}(q) \sin ^{2} \alpha+\sigma_{\mathrm{T}}(q)\left(1+\cos ^{2} \alpha\right)+I_{\mathrm{bg}}(q),
$$

where $\alpha$ is the angle $(\mathbf{q}, \mathbf{H})$, and $\sigma_{\mathrm{L}}(q)$ and $\sigma_{\mathrm{T}}(q)$ are the magnetic scattering cross sections related to correlations between transverse and longitudinal spin components, respectively. $I_{b g}(\mathrm{q})$ is an isotropic background, which consists of a low$q$ contribution from crystal inhomogeneities and a constant term, which can be calculated exactly, and which is in excellent agreement with experiment (see details in Ref. [48]).
Noticing that Eq. (1) fits the angular dependence of the intensity, we average the scattering map within two angular sectors of $60^{\circ}$ : sector 1 for $\mathbf{q} \| \mathbf{H}\left(\alpha=0^{\circ}\right)$ and sector 2 for $\mathbf{q} \perp \mathbf{H}\left(\alpha=90^{\circ}\right)$ [see Figs. 3(a)-3(c)]. We then combine the intensities from the two sectors to separate the contributions from the transverse and the longitudinal spin components [Fig. 3(d)].

As a key result, the intensity from the transverse spin components $\sigma_{\mathrm{T}}(q)$ shows a clear maximum in $q$, which arises from the vortexlike textures. As shown below, the FM correlated transverse spin components rotate over a finite length scale to compensate the transverse magnetization, yielding negligible intensity at $q=0$ and a maximum related to the vortex size. When the field increases, the maximum intensity decreases and its position moves towards high- $q$ values [Fig. 4(b)]. A signal from the transverse spin components is observed up to the highest field of $8 \mathrm{~T}$. On the other hand, the intensity from the longitudinal spin components $\sigma_{\mathrm{L}}(q)$ shows no welldefined maximum at $q \neq 0$ [Fig. 4(a)]. Above $2 \mathrm{~T}$, it becomes very small and difficult to separate from the background contribution [48]. 

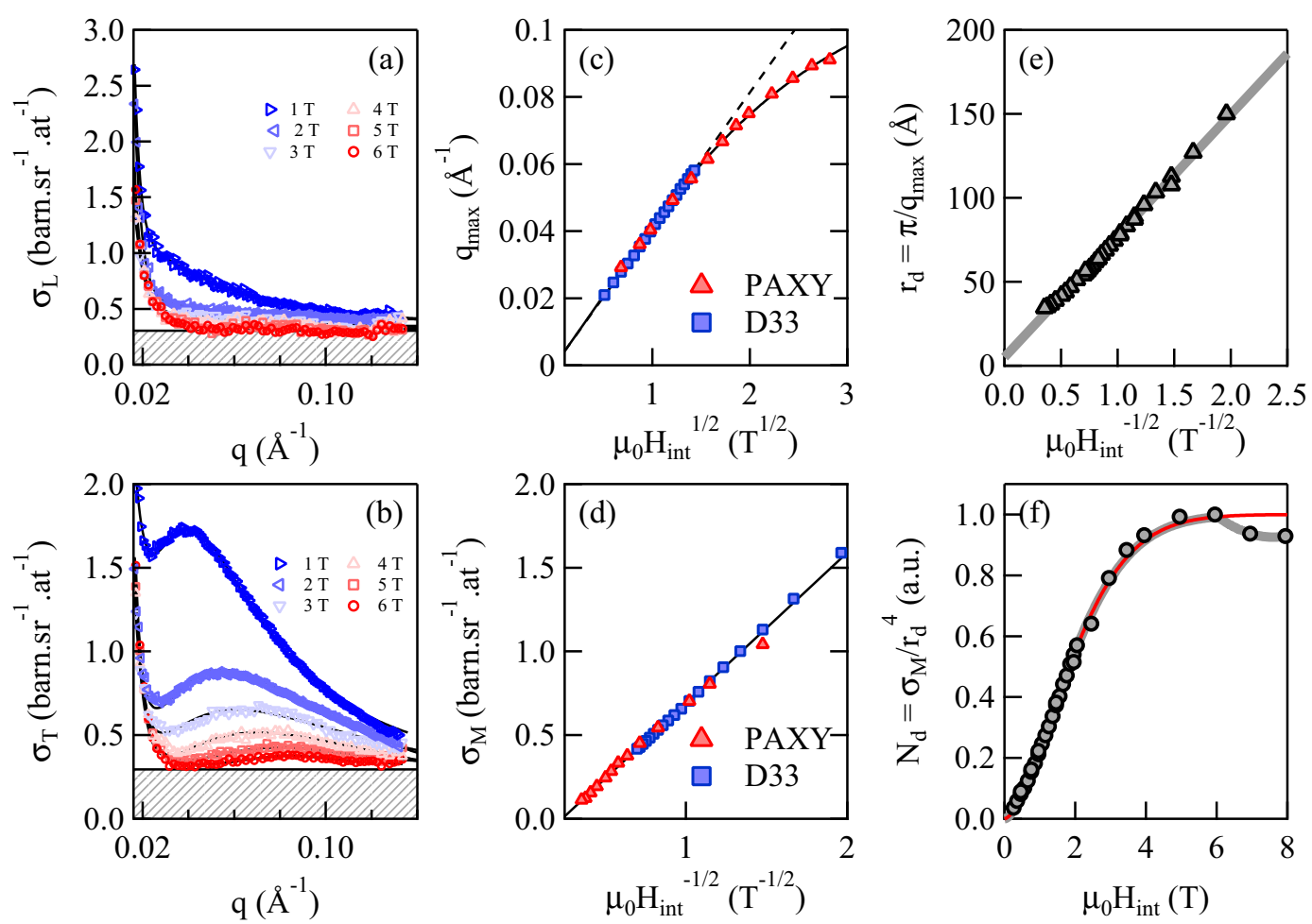

FIG. 4. Evolution of the longitudinal (a) and transverse (b) magnetic neutron scattering cross sections as a function of magnetic field. In both panels, the hatched areas represent the $q$-independent background contribution (see text and Ref. [48]). Field dependence of the peak position $q_{\max }(\mathrm{c})$ and integrated scattering intensity $\sigma_{\mathrm{M}}(\mathrm{d})$ as obtained from a fit of Eq. (2) to the SANS data from D33 (blue squares) and PAXY (red triangles). (e) Field dependence of the defect size $r_{\mathrm{d}}$, computed using data of (c). (f) Field dependence of the number of scattering centers $N_{\mathrm{d}}$, as seen by SANS. The red line is a fit of Eq. (4) to the data, in the $0 \leqslant \mu_{0} H_{\text {int }} \leqslant 6 \mathrm{~T}$ range.

In a first step, the transverse cross section was fitted by the phenomenological expression

$$
\begin{aligned}
\sigma_{T}(q)= & \frac{\sigma_{\mathrm{M}} \kappa q}{2 \pi q_{0}}\left(\frac{1}{\kappa^{2}+\left(q-q_{0}\right)^{2}}-\frac{1}{\kappa^{2}+\left(q+q_{0}\right)^{2}}\right) \\
& +\frac{I_{\mathrm{bg}}(q)}{2},
\end{aligned}
$$

where the first term accounts for the observed peak in the scattering cross section while the second one is related to the background. From Eq. (2), one can extract the peak position $q_{\max }=\sqrt{q_{0}^{2}+\kappa^{2}}$ and the integrated cross section $\sigma_{\mathrm{M}}$. As shown in Figs. 4(c)-4(f), these quantities vary continuously with the magnetic field.

To interpret these results, we take into account the liquidlike order of the defects in analogy with chemical inhomogeneities. Having fitted and subtracted the background term, we express the scattering cross section as

$\sigma_{T}(q)=a \Delta \rho_{\mathrm{mag}}^{2} N_{\mathrm{d}} V_{\mathrm{d}}^{2}\left\{\left\langle F_{\mathrm{T}}^{2}(q)\right\rangle-\left\langle F_{T}(q)\right\rangle^{2}\left[1-S_{\mathrm{int}}(q)\right]\right\}$,

where $F_{\mathrm{T}}(q)$ is the normalized form factor of the defects, associated with transverse spin components, and $S_{\text {int }}(q)$ is an interference function that takes into account the local correlations between two defects. In Eq. (3), $\langle\ldots\rangle$ denotes the statistical average over the sample. $\Delta \rho_{\mathrm{mag}} \simeq\left|\mathbf{m}_{\mathrm{T}}\right|$ is the magnetic contrast between a vortex (where $\left|\mathbf{m}_{\mathrm{T}}\right| \neq 0$ ) and the surrounding ferromagnetic region (where $\left|\mathbf{m}_{\mathrm{T}}\right| \rightarrow 0$ ). $N_{\mathrm{d}}$ and
$V_{\mathrm{d}}$ are, respectively, the number of vortices and their volume, and $a$ is a constant.

In the following, we neglect the local magnetic interaction between defects. This assumption of independent objects is justified for a weakly frustrated system where the vortex centers are randomly distributed and located far away from each other [i.e., $S_{\text {int }}(q)=1$ in Eq. (3)]. This assumption also holds for a system with concentrated defects, taking into account the specific form factor of the magnetic vortices and the random orientation of the transverse spin components from one vortex to another [i.e., $\left\langle F_{T}(q)\right\rangle=0$ in Eq. (3)]. It is confirmed by analytical calculations of model form factors [48] and by MC simulations reported in Sec. IV.

For independent defects, the $q$ dependence of the neutron intensity reduces to that of the average squared form factor, and the position $q_{\max }$ of the intensity maximum is inversely proportional to the typical size of the vortices. The integrated intensity $\sigma_{\mathrm{M}}$ is proportional to $\Delta \rho_{\mathrm{mag}}^{2} N_{\mathrm{d}} V_{\mathrm{d}}^{2}$, according to Eq. (3). As a toy model, we have considered regular vortices of radius $r_{\mathrm{d}}$ having an antiferromagnetic core [48]. The squared form factor averaged over all orientations for the transverse components has a nonsymmetric line shape recalling the experimental one, with a maximum at $q_{\max }=\pi / r_{\mathrm{d}}$.

Therefore, taking into account corrections for the demagnetization factor, the field dependence of $q_{\max }$ reflects the decrease of the vortex typical radius $r_{\mathrm{d}}=\pi / q_{\max }$ with increasing field [48]. Over the explored field range, $r_{\mathrm{d}}$ obeys the simple relation $r_{\mathrm{d}} \propto H^{-1 / 2}$ [Fig. 4(e)]. The corresponding variation of $\sigma_{\mathrm{M}} \propto H^{-1 / 2}$ suggests that the evolution of the 

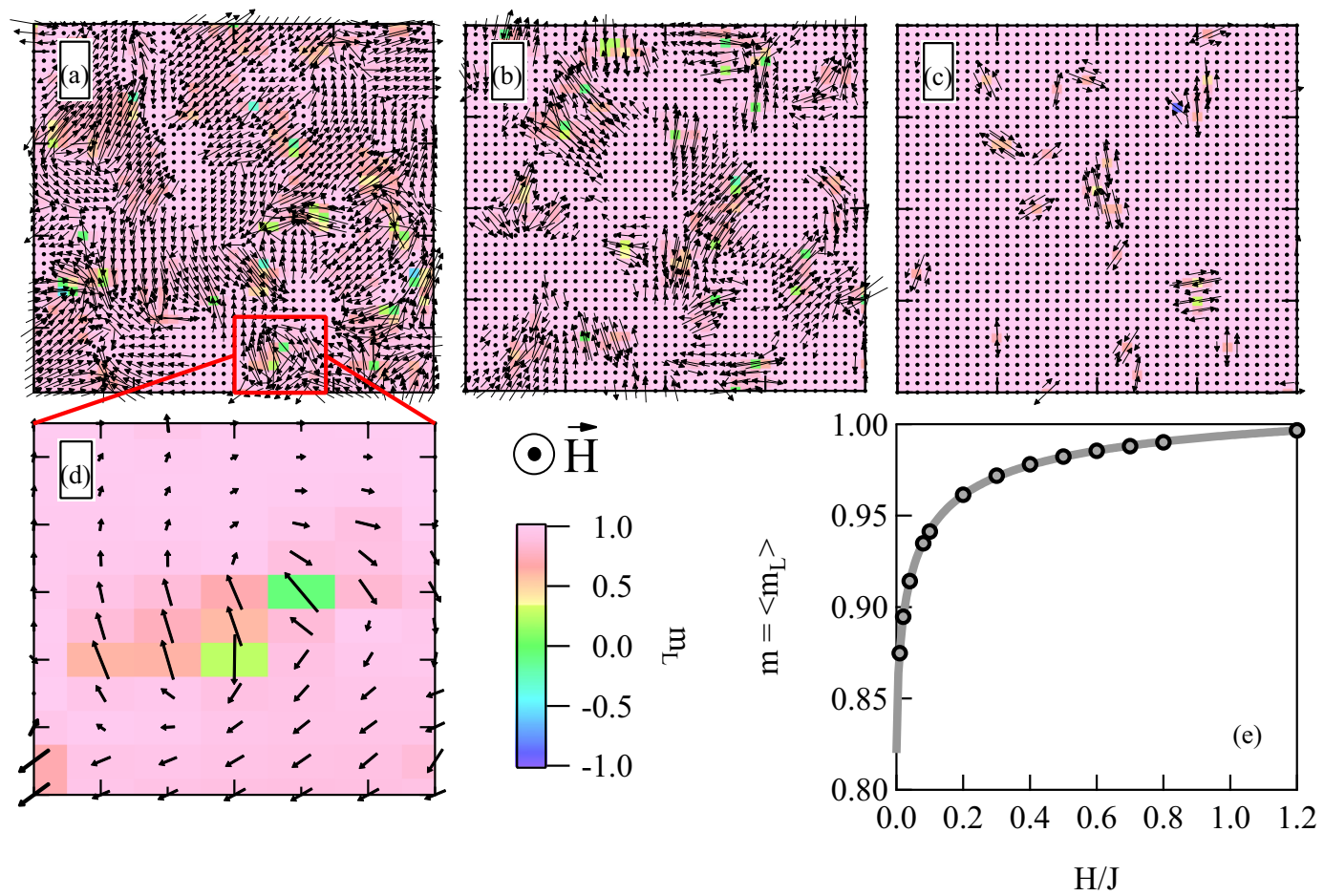

FIG. 5. Outcome of the Monte Carlo simulations for an antiferromagnetic bond concentration of 5\%. [(a)-(c)] Simulated spin maps for H/J $=0.01,0.1$, and 0.8 , respectively, where $\mathrm{J}$ is the first-neighbor exchange term. We display portions of $40 \times 40$ spins for clarity. The longitudinal magnetization $m_{\mathrm{L}}$ (i.e., parallel to the applied field) is color-coded while the arrows represent the transverse component $m_{\mathrm{T}}$. (d) Example of a vortexlike chiral spin circulation stabilized at $\mathrm{H}=0.01 \mathrm{~J}$. (e) Field dependence of the bulk magnetization $m$, defined as the average value of the longitudinal magnetization per spin $\left\langle m_{\mathrm{L}}\right\rangle$.

defect shape versus the magnetic field occurs in a self similar way, yielding scaling laws for the position, width and intensity of the magnetic signal [Fig. 4(b)]. Such laws are actually quite general and, for instance, govern the evolution of the cluster size with annealing time in metallic alloys which tend to segregate when they are quenched in the region of spinodal decomposition [51].

Using Eq. (3), one can also infer the field dependence of the number of defects (i.e., scattering centers) seen by SANS from the quantity $\sigma_{\mathrm{M}} / V_{\mathrm{d}}^{2}$. For this purpose, we assume thin cylindrical defects, and consider an experimental field range $H \ll J$ where the magnetic contrast (or amplitude of the transverse spin component) is roughly field-independent. We obtain $V_{\mathrm{d}} \simeq r_{\mathrm{d}}^{2}$, thus $N_{\mathrm{d}} \simeq \sigma_{\mathrm{M}} / r_{\mathrm{d}}^{4}$. As shown in Fig. 4(f), $N_{\mathrm{d}}$ increases with increasing field and saturates at a finite field of $\simeq 6 \mathrm{~T}$. This variation is described by a stretched exponential

$$
N_{\mathrm{d}}=1-\exp \left(-\frac{H}{H_{\mathrm{C}}}\right)^{v}
$$

with $H_{\mathrm{C}}=2.29(3) \mathrm{T}$ and $v=1.64(4)$. This result can be understood as follows. At low fields, vortices are large enough to involve several AFM bonds. Upon an increase in field, they progressively shrink while remaining centered on isolated AFM first-neighbor Mn-Mn pairs [48], the number of which is fixed by the Mn concentration and heat treatment. Consequently, the number of individual defects $N_{\mathrm{d}}$ seen by SANS will increase. At higher fields, however, $N_{\mathrm{d}}$ should decrease until all defects have collapsed for fields strong enough to overcome the typical AFM exchange interaction. We indeed observe a slight decrease of $N_{\mathrm{d}}$ for $\mu_{0} H_{\text {int }} \gtrsim 6 \mathrm{~T}$. However, we note that the field corresponding to the exchange interaction is of the order of several $100 \mathrm{~T}$ and is thus well-beyond our experimental range. In turn, this regime can be conveniently explored numerically. This point is addressed in the next section, where we propose a way to verify the above scenario and extend the exploration of the vortexlike textures properties towards arbitrarily large magnetic fields.

\section{MONTE CARLO SIMULATIONS}

Numerical studies of the reentrance phenomena and magnetic structures of reentrant spin glasses trace back to the pioneering work of Kawamura and Tanemura [26,52]. They showed that a minimal model is able to reproduce the main characteristics of the magnetic textures observed in RSG's. Following their approach, we first performed MC simulations on $2 \mathrm{~d}$ matrices containing $160 \times 160$ Heisenberg spins placed on a square lattice. While the main interaction is assumed to be FM $(J=1)$, a certain fraction $c$ of the bonds is turned into $\operatorname{AFM}(J=-1)$. Using a spin quench algorithm, the system ground state is found where vortexlike defects appear as metastable configurations (i.e., with energies slightly higher than those of the bulk FM state). For the studied concentrations $c=5 \%$ and $20 \%$, individual defects (similar to vortices or pairs of vortices) are evidenced, all of them being centered around the randomly distributed AFM NN pairs (see Fig. 5 for the 5\% case and Ref. [48] for further details). 

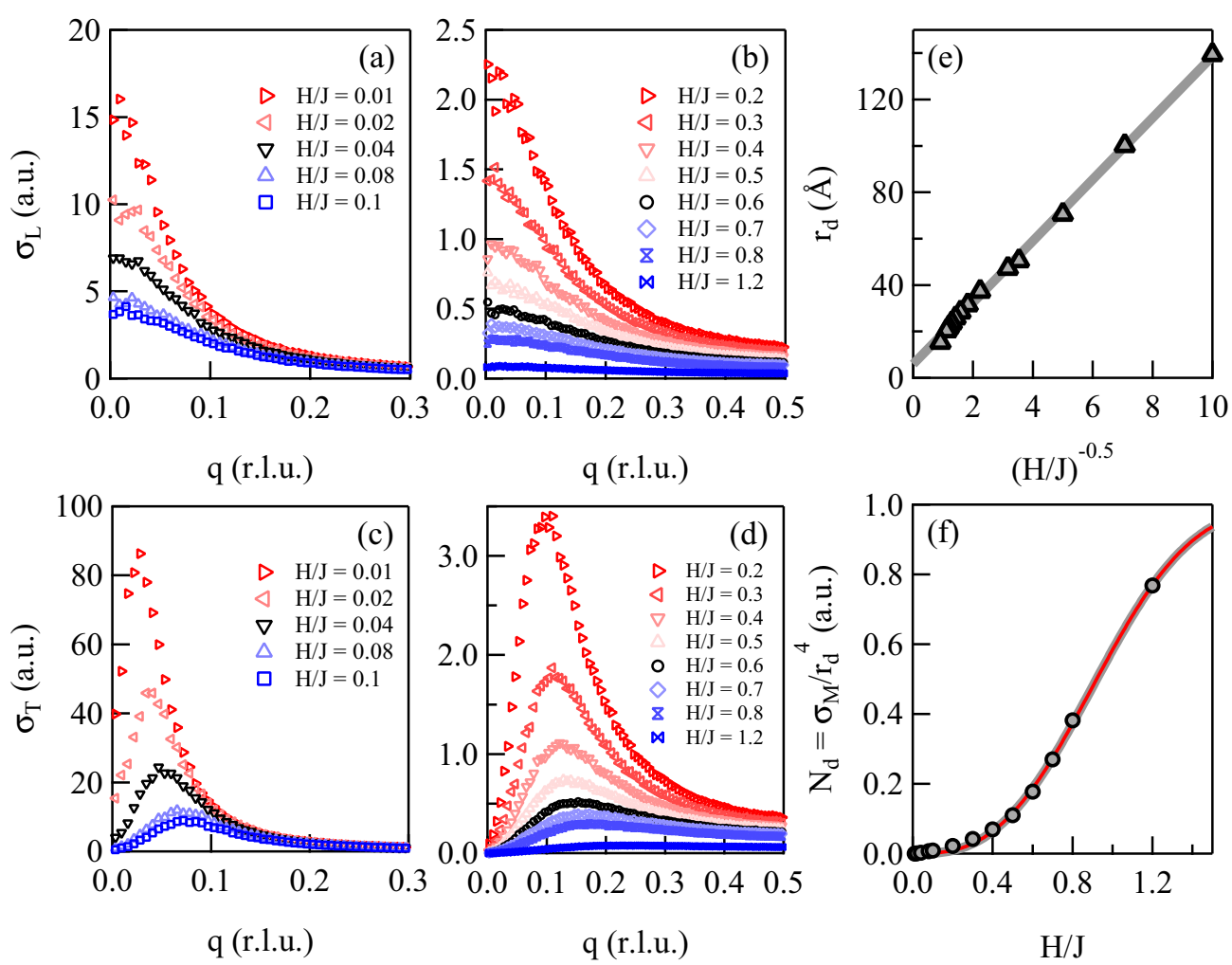

FIG. 6. Fourier analysis of the Monte Carlo results of Fig. 5. [(a)-(d)] Computed longitudinal [(a) and (b)] and transverse [(c) and (d)] form factors, obtained by Fourier transforming the calculated spin maps for different field values. The momentum $q$ is expressed in reciprocal lattice units (r.l.u.). (e) Field dependence of the average defect size $r_{\mathrm{d}}=a / q_{\max }$, where $q_{\max }$ is the position of the peak in $\sigma_{\mathrm{T}}$ and $a=3.586 \AA$ is the lattice constant of $\mathrm{Ni}_{0.81} \mathrm{Mn}_{0.19}$. (f) Field dependence of the apparent number of defects $N_{\mathrm{d}}$. The red line is a fit of Eq. (4) to the data, with an inflection point at $H_{\mathrm{C}}=1.05(5) \mathrm{J}$ and a stretching exponent $v=2.8(1)$.

In all cases, the average topological charge is $Q=0$ but individual objects locally display a finite $Q$, being in some cases as large as 0.3 (i.e., similar values as those found for certain types of frustrated SKs [30]). The origin of the noninteger charge is clarified by considering the relatively small size of the defects as well as their irregular shapes and distorted magnetization profiles, related to the ill-defined boundaries between vortices and the ambient FM medium. In other words, the vortexlike textures stabilized under a field in RSGs feature both senses of the vector chirality, resulting in a smaller topological charge than in frustrated or chiral SKs (for which $Q= \pm 1$ ). Extending the MC simulations to a 3d spin matrix, it appears that the vortexlike textures keep their anisotropic shape (oblate along the field direction) in the $3 \mathrm{~d}$ lattice and can thus be dubbed as "croutons."

As shown qualitatively on the maps displayed in Figs. 5(a)5(c), the average number of defects decreases with increasing field $H$ while spins are progressively aligned along its direction. The computed magnetization $m$ [Fig. 5(e)] increases as the number of vortices decreases, showing a quasiplateau with finite slope versus the ratio $H / J$. At very high fields, of magnitude comparable to the exchange constant $J$, a prediction of the MC modeling is that the vortices should collapse individually, yielding microscopic plateaus of $m$, the amplitude of which is probably too small to be experimentally observed.

In order to compare these results with the SANS experiments of Sec. III, we have computed the Fourier transforms of the longitudinal and transverse spin components. The longitudinal cross section $\sigma_{\mathrm{L}}$ decreases monotonically with increasing $q$ [Figs. 6(a) and 6(b)], whereas the transverse cross section $\sigma_{\mathrm{T}}$ shows a broad asymmetric peak [Figs. 6(c) and 6(d)]. Both quantities become almost $q$-independent at large $q$ values. When the field increases, the magnitude of the two simulated cross sections decreases, and a fit of Eq. (2) to the simulated $\sigma_{\mathrm{T}}$ curves shows that the position of the maximum $q_{\max }$ moves towards larger values, whereas its integrated intensity $\sigma_{\mathrm{M}}$ decreases. This evolution reflects a decrease of the vortex size $r_{\mathrm{d}}$ with increasing $H$ according to a scaling law [Fig. 6(e)] and an apparent increase of the number of vortices $N_{\mathrm{d}}$ following Eq. (4) with fit parameters $H_{\mathrm{C}}=1.05(5) J$ and $v=2.8(1)$ [Fig. 6(f)]. Similar to the experimental case, $N_{\mathrm{d}}$ is defined as $N_{\mathrm{d}} \simeq \sigma_{\mathrm{M}} / r_{\mathrm{d}}^{4}$, where $r_{\mathrm{d}}=a / q_{\max }$ with $a$ the lattice constant of $\mathrm{Ni}_{0.81} \mathrm{Mn}_{0.19}$. As discussed below, these results show that a minimal model is able to capture the essential features of the observed textures.

\section{DISCUSSION}

\section{A. Spin textures in a reentrant spin glass: the "crouton" picture}

The MC simulations presented above strongly reflect the experimental observations, as shown by the following. (i) The shape of the magnetization curve with a finite slope at large fields [compare Figs. 1(c) and 5(e)]. (ii) The existence of defects over which the transverse magnetization is self- 
compensated, yielding a peak of $\sigma_{T}$ at a finite $q$ value. The asymmetric $q$ dependence of $\sigma_{T}$ is also reproduced, suggesting similar internal structures of the defects [compare Figs. 4(b), 6(c), and 6(d)]. (iii) The persistence of inhomogeneities of the magnetization at the scale of the vortex size, deep inside the RSG phase, as shown by the finite longitudinal cross section $\sigma_{L}$ centered around $q=0$ [compare Figs. 4(a), 6(a), and 6(b)]. (iv) The field dependence of the defect size $r_{\mathrm{d}}$ (obtained from the $q$ position of the peak in $\sigma_{T}$ ), obeying scaling laws $r_{\mathrm{d}} \propto H^{-\beta}$ with the same exponent $\beta=0.5$ [compare Figs. 4(e) and 6(e)]. (v) The field dependence of the number of individual defects $N_{\mathrm{d}}$, increasing as a function of field following the phenomelogical Eq. (4), before reaching saturation [compare Figs. 4(f) and 6(f)]. (vi) The robustness of the defects, surviving up to very large fields as compared with usual magnetic SKs (compare Figs. 4(f) and 6(f) and see Ref. [48] for a detailed discussion).

Therefore the simulations strongly support a description of the magnetic defects observed in $\mathrm{Ni}_{0.81} \mathrm{Mn}_{0.19}$ as "croutonlike" defects, induced by AFM Mn-Mn first-neighbor pairs, where the transverse spin components are ferromagnetically correlated and rotate to compensate the transverse magnetization. Their magnitude decreases from the vortex center to the surroundings to accommodate the average ferromagnetic medium. As discussed below, such defect shape is compatible with the interactions generally considered for the RSGs, although other defect textures could be in principle compatible with the experiment.

The main difference between the experiment and the MC simulations is the field value at which the number of individual defects $N_{\mathrm{d}}$ starts saturating ( $H \ll J$ and $\sim J$, respectively). This suggests that an accurate determination of their stability range requires a more complex modeling, which is wellbeyond the scope of the present work. Indeed, the experimental situation is complicated, involving different moments on $\mathrm{Ni}$ and $\mathrm{Mn}$ ions, three types of interactions, a $3 \mathrm{~d}$ lattice with high connectivity, a high concentration of magnetic species, and an atomic short-range order (SRO). Therefore many different local environments and moment values exist in the experimental system. Comparatively, the simulations are based on a very simple case, namely, a $2 \mathrm{~d}$ square lattice with a random distribution of AFM bonds involving a single exchange constant.

Despite these differences, we stress that the agreement between both approaches is surprisingly good. Let us outline several reasons for that. Firstly, the mean-field description, which identifies longitudinal and transverse spin components with different behaviors, is valid, as expected for weak frustration. The present sample behaves as a weakly frustrated ferromagnet (the ratio $\mathrm{T}_{\mathrm{F}} / \mathrm{T}_{\mathrm{C}} \simeq 0.07$ can be associated to an effective concentration of AFM bonds of $\simeq 0.07$ in mean field approximation [48]), although the concentration of firstneighbor isolated $\mathrm{Mn}-\mathrm{Mn}$ pairs is relatively high (in the 0.2-0.4 range depending on the amount of SRO). Experiments varying the degree of frustration through $\mathrm{Mn}$ content or heat treatment could check the validity of this description when approaching the critical point which separates RSG and SG phases.

Secondly, both methods involve a statistical average of different types of defects, which do not interact with each other, but all have a typical size governed by general stability equations. This typical size is dictated by the competition between ferromagnetic exchange $\left(E=J k^{2}\right)$ and Zeeman energy, and it is expected to vary as $k^{-1} \propto(J / H)^{0.5}$, hence $r_{\mathrm{d}} \propto H^{-0.5}$, as observed experimentally and in the simulation. Such a general law also controls the extension of Bloch walls [53] or soliton defects [54] among others.

Our findings also suggest that the $2 \mathrm{~d}$ lattice provides a relevant description of the real case due to the peculiar crouton shape, with much larger extension in the transverse plane than along the field axis. In 2d-XY antiferromagnets, spontaneous vortices are stabilized and undergo a Kozterlitz-Thouless transition with temperature, involving spontaneous symmetry breaking at a local scale [55-57]. The reentrant transitions have a different nature, but they also involve peculiar symmetry breaking below $\mathrm{T}_{\mathrm{K}}$ and $\mathrm{T}_{\mathrm{F}}$, associated to the Gabay-Toulouse and de Almeida-Thouless lines, respectively [8]. As a major consequence, the transverse spin freezing and emergence of vortices strongly impact the spin excitations. A softening of the spin wave stiffness $[21,51]$ occurs below $T_{K}$, recalling the anomalous sound velocity in glasses [58,59] and the spin wave softening in quasi-2d frustrated antiferromagnets [60]. It is followed by a further hardening of the spin waves below $\mathrm{T}_{\mathrm{F}}$.

\section{B. Vortexlike textures and skyrmions}

Among the various classes of spin textures [61], those studied here show clear differences with the Bloch-type skyrmions observed in bulk chiral ferromagnets, which are primarily induced by DM anisotropy in noncentrosymmetric lattices. Both occur in an average ferromagnetic medium, but the vortexlike textures probed in this study are stabilized at low temperatures, do not form a magnetic lattice, and can exist for any crystal symmetry or even in amorphous compounds. This is because their primary origin is the competition of (symmetric) exchange interactions combined with site disorder, rather than antisymmetric exchange. In frustrated systems, the role of the latter, yielding DM anisotropy of chiral nature, has been investigated both theoretically $[62,63]$ and experimentally [22]. DM interactions explain the macroscopic irreversibilities in spin glasses and RSGs [64], torque measurements and paramagnetic resonance. Under field cooling conditions, they induce an additional magnetic field of unidirectional nature, which explains the slight decrease of the vortex size in NiMn when the sample is field-cooled $[48,65]$. However, they play a minor role in the stabilization of the vortex state, as exemplified by the MC results which describe a bare Heisenberg system. Experimentally, we point out that across the critical concentration, the vortices disappear in the true spin glass phase, while the DM anisotropy hardly changes [66].

The dissimilarities between vortices and frustrated SKs are more subtle but can be understood by a comparative analysis of their internal structure and topological charge, as deduced from MC simulations. This comparison is made in details in Ref. [48], and its main results are shown in Fig. 7. Essentially, frustrated SKs are predicted in ordered anisotropic magnets with competing interactions and inversion symmetry and do not require antisymmetric exchange [29-31]. Their center corresponds to a magnetic moment $\mathbf{m}$ antiparallel to the applied field $\left(m_{\mathrm{L}}<0\right)$, which gradually rotates towards the aligned state at the boundary $\left(m_{\mathrm{L}}>0\right)$. Therefore they 

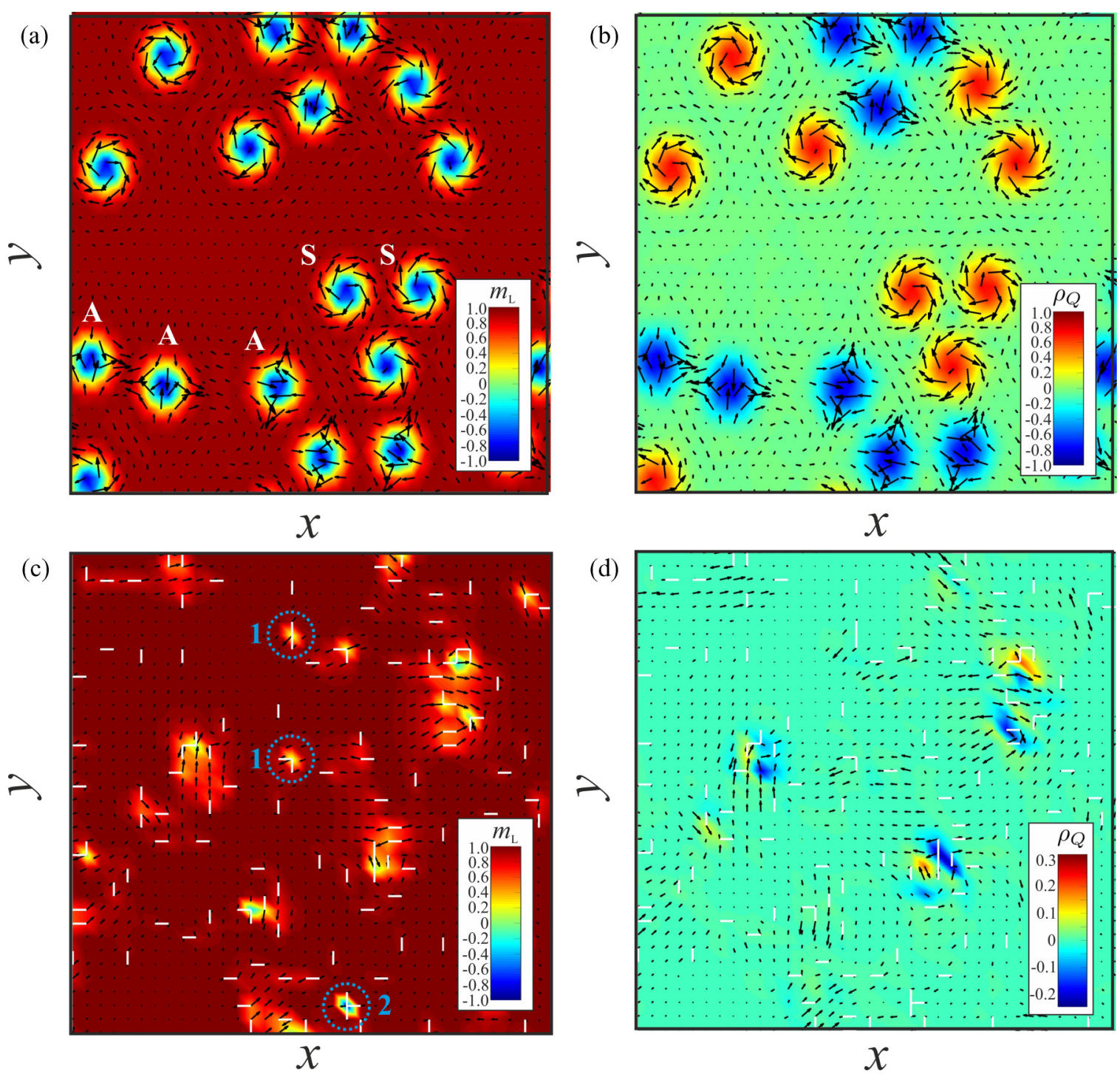

FIG. 7. [(a) and (b)] Skyrmions (S) and antiskyrmions (A) in a frustrated ferromagnet with competing NN and NNN exchange interactions $J_{1}$ and $J_{2}$ under a field $h$, with $J_{2} / J_{1}=0.5, h=0.4$. Typical metastable states featuring clusters of skyrmions and antiskyrmions are obtained by relaxing the magnetic configuration with a random initial spin configuration. Color plots of $m_{L}$ components (a) and the topological charge density $\rho_{Q}$ (b) reflect the smooth rotation of the magnetization within the skyrmion cores. [(c) and (d)] Vortexlike defects induced by interaction disorder as described by a model with random interaction $J_{i j}$ between $i, j$ sites under a field $h$. The $J_{i j}$ are independent random variables taking the values +1 and -1 with probabilities $1-c$ and $c$, respectively, with $c=0.05$ and $h=0.09$. Color plots of $m_{L}$ components (c) exhibit various types of spatially localized objects with the balanced topological charge density (d). Blue numbered circles show vortices residing on two or three AFM bonds with distinct magnetization distributions. See Ref. [48] for details.

individually possess a large topological charge $(+1$ or -1$)$ and can form densely packed clusters. These features contrast with the vortexlike textures studied in this work, pinned by locally disordered AFM bonds and unable to form extended ordered phases. Since $m_{\mathrm{L}}$ can alternate in sign in the vortex core (as controlled by their internal bond structure), the vortices neither bear smooth rotation of $\mathbf{m}$ nor select a preferred helicity, and their absolute topological charge density is smaller than unity within isolated defects.

Although they cannot form ordered phases, the vortices studied in the present work may form a liquidlike order in the limit of small applied magnetic fields and large concentration of AFM bonds. Finally, both vortices and frustrated SKs remain metastable solutions, found at zero or finite temperatures. They are both endowed with a remarkable robustness against the collapse towards the field-induced FM state and do not require well-defined lattice structures to appear.

\section{OUTLOOK AND CONCLUSION}

Our study suggests that in the general search for SK-hosting systems, the role of disorder should be investigated more intensively. Its main consequences are expected in the low energy dynamics, associated with glassy states which occur both in average ordered or disordered media. Theoretically, the glassy behavior is related to metastable states with hierarchical structure in the ground-state manifold. It can be analyzed in terms of replica field theory, initially developed 
for spin glasses [7], then extended to RSGs [8], vortex lattices in superconductors (the Bragg glass phases [67]), and very recently to the skyrmion glass phase [36]. The example of Co-Zn-Mn alloys [37] where SK lattices are observed at $300 \mathrm{~K}$ and above, is an interesting playground to study such aspects in detail. There, site inversions should lead to local frustration effects and possibly explain the metastable textures observed experimentally.

Our work constitutes an experimental illustration of the importance of frustration and disorder for the emergence of localized spin textures in condensed matter. We suggest a simple mechanism for tuning their properties (density, size) by different parameters such as the magnetic field, heat treatment, or concentration. This could open a promising route towards the engineering of bulk systems with well defined sizes and density ranges, for instance, the design of vortices by a controllable distribution of bonds. Moreover, while the observed vortices cannot be moved since they are bound to the Mn-Mn pairs, their interaction with electric currents $[68,69]$ and spin waves [70-72] is nontrivial. In both cases, the class of frustrated ferromagnets studied in this paper might offer novel ways to encode complex information into electron and heat pulses.

\section{ACKNOWLEDGMENTS}

We thank P. Bonville for the SQUID measurements, M. Bonnaud for technical assistance on the D33 spectrometer and S. Gautrot and V. Thevenot for their help in setting the experiment on PAXY. A.O.L. acknowledges JSPS Core-toCore Program, Advanced Research Networks (Japan) and JSPS Grant-in-Aid for Research Activity Start-up 17H06889, and thanks Ulrike Nitzsche for technical assistance.
[1] K. v. Klitzing, G. Dorda, and M. Pepper, Phys. Rev. Lett. 45, 494 (1980).

[2] D. J. Thouless, M. Kohmoto, M. P. Nightingale, and M. den Nijs, Phys. Rev. Lett. 49, 405 (1982).

[3] A. Fert and I. A. Campbell, Phys. Rev. Lett. 21, 1190 (1968).

[4] M. N. Baibich, J. M. Broto, A. Fert, F. Nguyen Van Dau, F. Petroff, P. Etienne, G. Creuzet, A. Friederich, and J. Chazelas, Phys. Rev. Lett. 61, 2472 (1988).

[5] P. Le Doussal and T. Giamarchi, Phys. Rev. B 57, 11356 (1998).

[6] T. Klein, I. Joumard, S. Blanchard, J. Marcus, R. Cubitt, T. Giamarchi, and P. Le Doussal, Nature 413, 404 (2001).

[7] D. Sherrington and S. Kirkpatrick, Phys. Rev. Lett. 35, 1792 (1975).

[8] M. Gabay and G. Toulouse, Phys. Rev. Lett. 47, 201 (1981).

[9] W. Abdul-Razzaq and J. S. Kouvel, J. Appl. Phys. 55, 1623 (1984).

[10] I. A. Campbell, S. Senoussi, F. Varret, J. Teillet, and A. Hamzić, Phys. Rev. Lett. 50, 1615 (1983).

[11] K. Motoya, S. M. Shapiro, and Y. Muraoka, Phys. Rev. B 28, 6183 (1983).

[12] P. Böni, S. Shapiro, and K. Motoya, Solid State Commun. 60, 881 (1986).

[13] S. K. Burke and B. D. rainford, J. Phys. F 13, 441 (1983).

[14] M. B. Salamon, K. V. Rao, and H. S. Chen, Phys. Rev. Lett. 44, 596 (1980).

[15] R. J. Birgeneau, J. A. Tarvin, G. Shirane, E. M. Gyorgy, R. C. Sherwood, H. S. Chen, and C. L. Chien, Phys. Rev. B 18, 2192 (1978).

[16] J. A. Fernandez-Baca, J. J. Rhyne, G. E. Fish, M. Hennion, and B. Hennion, J. Appl. Phys. 67, 5223 (1990).

[17] S. Senoussi, S. Hadjoudj, P. Jouret, J. Bilotte, and R. Fourmeaux, J. Appl. Phys. 63, 4086 (1988).

[18] S. Senoussi, S. Hadjoudj, R. Fourmeaux, and C. Jaouen, J. Phys. Colloques 49, C8-1163 (1988).

[19] M. Hennion, I. Mirebeau, B. Hennion, S. Lequien, and F. Hippert, Europhys. Lett. 2, 393 (1986).

[20] S. Lequien, I. Mirebeau, M. Hennion, B. Hennion, F. Hippert, and A. P. Murani, Phys. Rev. B 35, 7279 (1987).
[21] M. Hennion, B. Hennion, I. Mirebeau, S. Lequien, and F. Hippert, J. Appl. Phys. 63, 4071 (1988).

[22] I. A. Campbell, H. Hurdequint, and F. Hippert, Phys. Rev. B 33, 3540 (1986).

[23] G. Tatara and H. Kawamura, J. Phys. Soc. Jpn. 71, 2613 (2002).

[24] P. Pureur, F. W. Fabris, J. Schaf, and I. A. Campbell, Europhys. Lett. 67, 123 (2004).

[25] F. W. Fabris, P. Pureur, J. Schaf, V. N. Vieira, and I. A. Campbell, Phys. Rev. B 74, 214201 (2006).

[26] H. Kawamura and M. Tanemura, J. Phys. Soc. Jpn. 60, 1092 (1991).

[27] A. Fert, V. Cros, and J. Sampaio, Nat. Nano 8, 152 (2013).

[28] N. Nagaosa and Y. Tokura, Nat. Nano. 8, 899 (2013).

[29] T. Okubo, S. Chung, and H. Kawamura, Phys. Rev. Lett. 108, 017206 (2012).

[30] A. O. Leonov and M. Mostovoy, Nat. Commun. 6, 8275 (2015).

[31] S.-Z. Lin and S. Hayami, Phys. Rev. B 93, 064430 (2016).

[32] L. Rózsa, A. Deák, E. Simon, R. Yanes, L. Udvardi, L. Szunyogh, and U. Nowak, Phys. Rev. Lett. 117, 157205 (2016).

[33] Y. Hu, X. Chi, X. Li, Y. Liu, and A. Du, Sci. Rep. 7, 16079 (2017).

[34] A. O. Leonov, T. L. Monchesky, N. Romming, A. Kubetzka, A. N. Bogdanov, and R. Wiesendanger, New J. Phys. 18, 065003 (2016).

[35] A. O. Leonov, Y. Togawa, T. L. Monchesky, A. N. Bogdanov, J. Kishine, Y. Kousaka, M. Miyagawa, T. Koyama, J. Akimitsu, T. Koyama et al., Phys. Rev. Lett. 117, 087202 (2016).

[36] S. Hoshino and N. Nagaosa, Phys. Rev. B 97, 024413 (2018).

[37] Y. Tokunaga, X. Z. Yu, J. S. White, H. M. Rønnnow, D. Morikawa, Y. Taguchi, and Y. Tokura, Nat. Commun. 6, 7638 (2015).

[38] A. K. Nayak, V. Kumar, T. Ma, P. Werner, E. Pippel, R. Sahoo, F. Damay, U. K. Rößler, C. Felser, and S. S. P. Parkin, Nature (London) 548, 561 (2017).

[39] T. Kurumaji, T. Nakajima, M. Hirschberger, A. Kikkawa, Y. Yamasaki, H. Sagayama, H. Nakao, Y. Taguchi, T. Arima, and Y. Tokura, arXiv:1805.10719.

[40] R. Sommer, J. Schmidt, and A. Gomes, J. Magn. Magn. Mater. 103, 25 (1992). 
[41] W. Abdul-Razzaq and J. S. Kouvel, Phys. Rev. B 35, 1764 (1987).

[42] I. Mirebeau, Ph.D. thesis, Université de Paris-Sud, 1987.

[43] M. J. Marcinkowski and N. Brown, J. Appl. Phys. 32, 375 (1961).

[44] J. W. Cable and H. R. Child, Phys. Rev. B 10, 4607 (1974).

[45] Y. Yokoyama and T. Satoh, J. Magn. Magn. Mater. 3, 347 (1976).

[46] T. Okazaki, Jpn. J. Appl. Phys. 34, 1537 (1995).

[47] G. J. Stanisz, J. Soltys, and J. M. Holender, J. Phys.: Condens. Matter 1, 6327 (1989).

[48] See Supplemental Material at http://link.aps.org/supplemental/ 10.1103/PhysRevB.98.014420 for details about data treatment, calculations of neutron scattering cross sections, additional SANS data, mean-field modeling of the NiMn alloy and a comparative analysis of the structure of vortexlike defects and frustrated skyrmions.

[49] J. W. Lynn, N. Rosov, T. E. Grigereit, H. Zhang, and T. W. Clinton, Phys. Rev. Lett. 72, 3413 (1994).

[50] S. Mühlbauer, B. Binz, F. Jonietz, C. Pfleiderer, A. Rosch, A. Neubauer, R. Georgii, and P. Böni, Science 323, 915 (2009).

[51] M. Hennion, D. Ronzaud, and P. Guyot, Acta Metall. 30, 599 (1982).

[52] H. Kawamura and M. Tanemura, J. Phys. Soc. Jpn. 55, 1802 (1986).

[53] G. T. Rado, Phys. Rev. B 26, 295 (1982).

[54] M. Steiner, J. Magn. Magn. Mater. 31-34, 1277 (1983).

[55] J. M. Kosterlitz and D. J. Thouless, J. Phys. C 6, 1181 (1973).
[56] J. M. Kosterlitz, Rev. Mod. Phys. 89, 040501 (2017).

[57] J. Villain, J. Phys. C 36, 581, (1975).

[58] J. L. Black and B. I. Halperin, Phys. Rev. B 16, 2879 (1977).

[59] K. H. Michel, Phys. Rev. B 35, 1414 (1987).

[60] D. N. Aristov and S. V. Maleyev, Z. Phys. B 81, 433 (1990).

[61] G. Toulouse and M. Kléman, J. Phys. Lett. 37, 149 (1976).

[62] C. M. Soukoulis, G. S. Grest, and K. Levin, Phys. Rev. Lett. 50, 80 (1983).

[63] M. J. P. Gingras, Competition Between Ferromagnetic and Spin Glass Order in Random Magnets: The Problem of Reentrant Spin Glasses (World Scientific, Singapore, 1994), pp. 238-285.

[64] S. Senoussi, Phys. Rev. Lett. 51, 2218 (1983).

[65] I. Mirebeau, M. Hennion, S. Lequien, and F. Hippert, J. Appl. Phys. 63, 4077 (1988).

[66] N. Martin et al. (unpublished).

[67] T. Giamarchi and P. Le Doussal, Phys. Rev. B 52, 1242 (1995).

[68] D. Prychynenko, M. Sitte, K. Litzius, B. Krüger, G. Bourianoff, M. Kläui, J. Sinova, and K. Everschor-Sitte, Phys. Rev. Appl. 9, 014034 (2018).

[69] G. Bourianoff, D. Pinna, M. Sitte, and K. Everschor-Sitte, AIP Adv. 8, 055602 (2018).

[70] M. Continentino, J. Magn. Magn. Mater. 31-34, 1413 (1983).

[71] I. Y. Korenblit, S. V. Maleev, and E. F. Shender, Phys. Rev. B 33, 624 (1986).

[72] E. F. Shender and R. Rammal, Europhys. Lett. 15, 795 (1991). 